\title{
Geringe funktionelle Besserung ohne Einfluss auf die Lebensqualität
}

Fragestellung: Sind orale Kortikosteroide zur Behandlung radikulärer Schmerzen bei lumbalen Bandscheibenvorfällen wirksam?

Hintergrund: Die Lebenszeitprävalenz von radikulären Schmerzen bei lumbosakralen Bandscheibenvorfällen beträgt $10 \%$. Sehr häufig kommt es zu einer spontanen Remission. Bis dahin haben die Patienten zum Teil allerdings erhebliche Schmerzen. Nicht steroidale Antirheumatika (NSAR) sind in dieser Situation wirksam, während Paracetamol in mehreren randomisierten placebokontrollierten Studien nicht wirksam war. Eine Reihe von randomisierten Studien haben in letzter Zeit gezeigt, dass die lokale Injektion von Lokalanästhetika und/oder Kortikosteroiden einer Placeboinjektion für langfristige Therapieerfolge nicht überlegen ist. Viele Neurologen und Schmerztherapeuten verwenden allerdings bei länger anhaltenden radikulären Schmerzen bei Bandscheibenvorfällen Kortikosteroide entweder als Monotherapie oder zusätzlich zu NSAR.

Patienten und Methodik: Die randomisierte, doppelblinde, placebokontrollierte Studie schloss erwachsene Patienten ein, die nicht länger als drei Monate unter radikulären Schmerzen mit einem Oswestry Disability Index Score von 30 oder hö-

Goldberg H, Firtch W, Tyburski $M$ et al. Oral steroids for acute radiculopathy due to a herniated lumbar disk: a randomized clinical trial. JAMA 2015; 313: $1915-23$ her (Bereich 0-100) und einem in der Kernspintomografie nachgewiesenen passenden Bandscheibenvorfall litten. Die Patienten wurden im Verhältnis 2:1 randomi- siert und erhielten entweder orales Prednison in absteigender Dosis über 15 Tage (60 mg, $40 \mathrm{mg}, 20 \mathrm{mg}$ ), Gesamtdosis $600 \mathrm{mg}$ oder Placebo. 181 Patienten erhielten Prednison und 88 Placebo. Der primäre Outcome war die Veränderung im Oswestry Disability Index Score nach drei Wochen. Sekundäre Endpunkte waren die Veränderung des Scores nach einem Jahr, die Schmerzintensität der radikulären Schmerzen auf einer Skala von 0 - 10, der SF 36 für die Lebensqualität, die Physical Component Summary und die Mental Component Summary.

Ergebnisse: Die Patienten waren im Mittel 46 Jahre alt (55\% Männer). Der Oswestry Disability Index Score bei Einschluss betrug 51. Die Schmerzintensität der radikulären Schmerzen wurde im Mittel mit 6,7 angegeben. Im Mittel vergingen 30 Tage zwischen Beginn der Schmerzsymptomatik und Randomisierung. Der Oswestry Disability Index Score verbesserte sich nach drei Wochen um 19 Punkte in der Prednison- und um 13 Punkte in der Placebogruppe. Dieser Unterschied war statistisch signifikant. Nach 52 Wochen hatte der Score um 37,8 Punkte in der Prednison- und um 30,4 in der Placebogruppe verbessert. Dieser Unterschied war ebenfalls signifikant. Bezüglich der radikulären Schmerzen ergab sich zu beiden Zeitpunkten kein Unterschied. Auch bezüglich der Häufigkeit späterer Bandscheibenoperationen ergab sich kein Unterschied. Auch für die Lebensqualität zeigten sich keine Unterschiede.

Schlussfolgerungen: Eine orale Prednisontherapie über einen Zeitraum von 15 Tagen ergibt im Vergleich zu Placebo eine geringe Besserung der funktionellen Einschränkung, hat aber keinen Einfluss auf die Schmerzintensität und die Lebensqualität.

\section{- Kommentar von Hans-Christoph Diener, Essen}

\section{Keine Auswirkungen auf Schmerzintensität und Lebensqualität}

Diese sehr gute und stringent durchgeführte Studie belegt einen geringen Effekt einer oralen Kortisontherapie auf den funktionellen Outcome nach drei Wochen und einem Jahr. Interessanterweise hat die Therapie aber keine Auswirkung auf die Schmerzintensität und die Lebensqualität. Durch die Therapie konnten auch spätere Operationen an den Bandscheiben nicht verhindert werden.

Für den klinischen Alltag würde dies bedeuten, dass Patienten, soweit keine Kontraindikationen bestehen, zunächst mit nicht steroidalen Antirheumatika und/oder retardierten Opioiden behandelt werden sollten. Wenn diese nicht ausreichend wirksam sind, kann dann im Einzelfall eine zeitlich befristete Kortisongabe durchgeführt werden. Der Nutzen von Kortison in der Kombinationstherapie wurde allerdings in der hier vorliegenden Studie nicht untersucht.

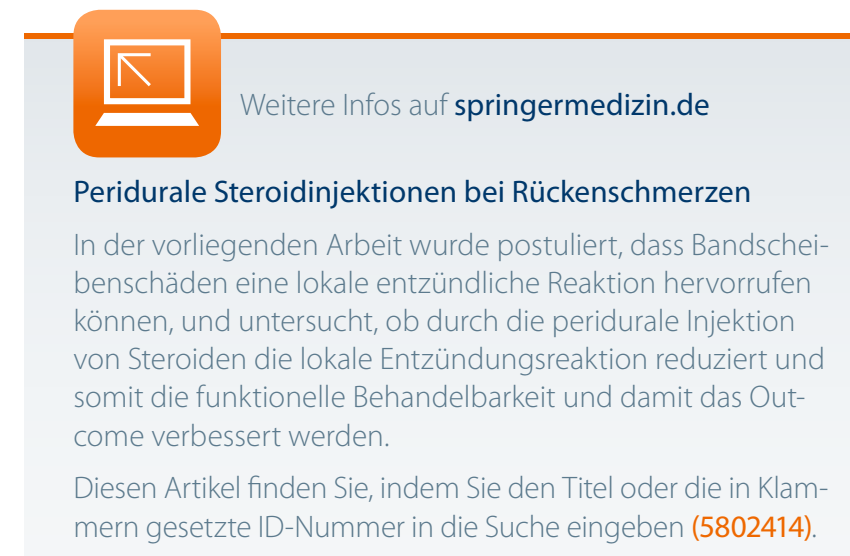

